\title{
REVIEW
}

\section{Current Update and Trends in Melanin Pigmentation and Melanin Biology}

\author{
Kowichi Jimbow \\ Department of Dermatology and Cutaneous Sciences, University of Alberta, Edmonton, Alta, Canada
}

(Reccived for publication on December 26, 1994)

\begin{abstract}
Certain aspects of current progress in melanin biology and its possible clinical relevances are reviewed with emphasis on some of our recent research activities. The important aspects discussed are (a) the biological properties of melanin pigments and their relevance to biological functions, (b) functional interaction of melanogenic/melanogenesis-associated genes in melanin biosynthesis process (melanogenesis, and (c) the targeting of proteins involved in melanogenesis and assembly by melanosomal proteins. Upon exposure to UV light radiation (UVR), melanin pigments revealed two distinct photobiological reactions, i.e. photoprotective and phototoxic reactions. The precursor intermediate of brown-black eumelanin, 5-6-dihydroxyindole, appears to possess the most potent photoprotective (antioxidant) property. Eumelanin pigment also had some antioxidant property. Similarly, yellow-red pheomelanin and its precursor intermediate, 5-S-cysteinyldopa also revealed some antioxidant property, but they became prooxidant in the presence of the ferric iron upon exposure to UVR. Melanosomes are known to possess several metal ions including $\mathrm{Fe}^{2+}, \mathrm{Fe}^{3+}, \mathrm{Cu}^{2+}$ and $\mathrm{Zn}^{2+}$. In addition, upon exposure to $\mathrm{UV}$-light, there is an increase in ferric/ferrous iron in the skin. Therefore, in the in vivo system, pheomelanin intermediate, 5-Scysteinyldopa, may show significant prooxidant property in conjunction with metal ions (e.g. $\mathrm{Fe}^{2+}, \mathrm{Fe}^{3+}$ ). When atypical moles (previously called dysplastic nevi) were analysed chemically for quantitative and qualitative properties of melanin pigment, they revealed a high ratio of pheomelanin/eumelanin content. This finding may partly explain our clinical observation that these moles are frequently the precursors of malignant melanoma and that the intermittent heavy exposure of UVR can be the major direct cause of their transformation. How, then, the melanosomal compartment, where active new melanin synthesis occurs after exposure to UVR, is protected from the cytotoxicity of melanin precursor intermediates. To study this question, two major experiments were conducted; (a) expression of melanogenesis-associated genes upon exposure of melanocytes to UVR and (b) transfection of cDNAs from the melanogenesisassociated genes. There was coordinated gene expression (mRNA) of tyrosinase and tyrosinase-related protein, TRP-1 and this coordinated gene expression was also accompanied by the upregulation of LAMP-1 (lysosome-associated membrane protein-1). Furthermore, human tyrosinase and TRP-1 mRNAs were expressed successfully in individual transfectants or co-transfectants of cDNAs from the two genes. Co-transfectants of human tyrosinase and TRP-1 cDNAs produced many lysosomal granules and melanin-containing granules, melanosomes. LAMP-1 gene was upregulated simultaneously in co-transfectants of tyrosinase and TRP-1, but not in individual transfectants of the two genes. TRP-1 and LAMP-1 gene products were transferred from Golgi complexes to melanosomes. LAMP-1 gene products stimulated by TRP-1 and tyrosinase genes may prevent programmed death of melanocytes due to cytotoxic melanin precursor intermediates by coating the inner surface of melanosomal membrane through the $\mathrm{N}$ - and $\mathrm{O}$-linked oligosaccharide moieties of LAMP-1 which have been suggested to protect lysosomes from the action of hydrolytic enzymes. (Keio J Med 44 (1): 9-18, March 1995)
\end{abstract}

Key words: melanogenesis, melanocyte, malignant melanoma, UV light

Presented at the 896th Meeting of The Keio Medical Society in Tokyo, December 8, 1994.

Reprint requests to: Dr Kowichi Jimbow, Division of Dermatology and Cutancous Sciences, 260G Heritage Medical Research Center, University of Alberta, Edmonton, Alberta, Canada, T6G 2S2 
Abbreviations: UVR; ultraviolet light radiation, TRP; tyrosinase-related protein, DHI; dihydroxyindole, DHICA; dihydroxyindolc-2-carboxylic acid, EPR; electron paramagnetic resonance, SCD; 5-S-cysteinyldopa, TMB: tetramethyl benzidine, MDA; malonedialdehyde, TBARS; thiobarburic acid reactive substances, LAMP; lysosome-associated membrane protein, HMSA: human melanosome specific antigen.

\section{Introduction}

Human melanocytes migrate from the embryonic neural-crest tissue into the skin and then produce unique secretory granules, melanosomes, which contain melanin pigments. Melanin and melanosomes are the major determinants of skin pigmentation. Melanin-forming enzyme tyrosinase is the major component responsible for melanogenesis, which occurs within the compartment of secretory granules, melanosomes.'

Mechanisms involved in the biosynthesis and structural organization of melanosomes are not well understood. An elucidation of the molecular events involved in these processes would be important in understanding the biology and pathophysiology of normal and abnormal pigmentation in the skin. The pathological role of pigmentation originates mainly from the fact that melanin pigments serve not only as the major determinant of the skin color but also as the major source of skin protectant against radiation, preventing sun-induced skin damage as well as skin cancer development. ${ }^{2}$ This elucidation may be equally important for an explanation as to why the melanosomal structures become markedly aberrant in various melanocytic disorders including neoplastic counterpart, malignant melanoma. ${ }^{3}$ Abnormal melanin pigmentation can also be the direct cause of a number of congenital and acquired pigmentary skin diseases. ${ }^{4}$ For example, abnormal degradation of melanosomes has been suggested to be a direct cause of programmed death of melanocytes of vitiligo and related diseases. ${ }^{5}$ These changes may result from abnormal expression of genes involved in the biosynthesis of melanin and melanosomes, which leads directly or indirectly to alteration in the structure and/or function of melanosomes. Disturbed rates of transcription and putative alternative splicing of mRNAs may also contribute to the melanosomal derangement which would lead to the formation of abnormal melanosomes seen in such diseases as von Recklinghausen's disease and Chediak-Higashi syndrome." This review introduces three major topics. The first topic deals with biological properties of melanin pigments, in particular, after exposure to UVR, and the second one addresses the functional interaction of tyrosinase and LAMP gene families in melanogenesis, using the cells exposed to UVR. Signals targeting the transport of proteins into the melanosomal compartment may be disturbed due to altered co- and/or posttranslational modification. A better understanding of such putative biological and physiological processes may best be obtained by isolating DNA probes for genes relevant to melanogenesis, transfecting these genes in either wild or mutated form into non-pigmented or amelanotic cells and correlating the expression of mRNAs and their products with cell growth and differentiation. The third topic, therefore, is the possibility of using the gene transfection experiments in understanding the target signals of melanogenic proteins and melanosomal assembly.

\section{Biological Properties of Melanin Pigments}

\section{$U V$ radiation and melanin pigmentation}

UV light can be the most potent stimulator for growth and differentiation of melanocytes. Melanocyte activation and differentiation can be induced directly by UV irradiation or indirectly through the interaction with UV-irradiated surrounding keratinocytes. For example, UV radiation can increase the synthesis of bFGF in keratinocytes which will then stimulate the melanogenesis of epidermal melanocytes. ${ }^{7.8}$ Similarly, MSH production by keratinocytes can be stimulated by UV radiation and also enhance the expression of MSH receptor on the melanocyte which will then increase the melanogenesis by the melanocyte. ${ }^{y}$ There are, of course, growth factors and regulators which are not directly related to UV exposure. For example, c-Kit which is a proto-oncogene encoding a membrane receptor protein of molecular weight of $145 \mathrm{kDa}$ with intrinsic tyrosine kinase activity in melanocytes. C-Kit gene expression is essential for the proper embryonic development of melanocytes (Fig 1). ${ }^{10}$ If there is any mutation of $\mathrm{c}-\mathrm{Kit}$, this could cause white spotting in mice and piebaldism in humans, affecting the proliferation and survival of melanocyte precursors during embryonic differentiation. The c-Kit expression would also be important in the proliferation of adult melanocytes and may also be partly related to their differentiation after exposure to UVR.

The melanocyte performs the unique metabolic activity of melanin synthesis. In a complex series of reactions occurring in specialized organelles, melanosomes, tyrosinase catalyzes the first two reactions of the biochemical pathway leading to the formation of two types of highly polymerized melanin pigments, eumelanin or pheomelanin (Fig 2). Tyrosinase catalyzes the hydroxylation of tyrosine to form dopa (L-3,4-dihydroxyphenylalanine) and then the oxidation of dopa to dopaquinone. The latter is rapidly converted to the brown-black pigment, eumelanin, via 5,6-dihydroxyindole (DHI) or dihydroxyindole-2-carboxylic acid (DHICA). However, intervention of sulphydryl compounds in melanogenesis leads, via 5-S-cysteinyldopa (SCD), to the formation of the yellow-red pigment, pheomelanin (Fig 3). Eumelanin is the major pigment in normal human skin, although small 
amounts of pheomelanin can be seen in certain ethnic groups (e.g. red-haired, freckled Caucasians). The exposure of human melanocytes to UV light or melanocyte

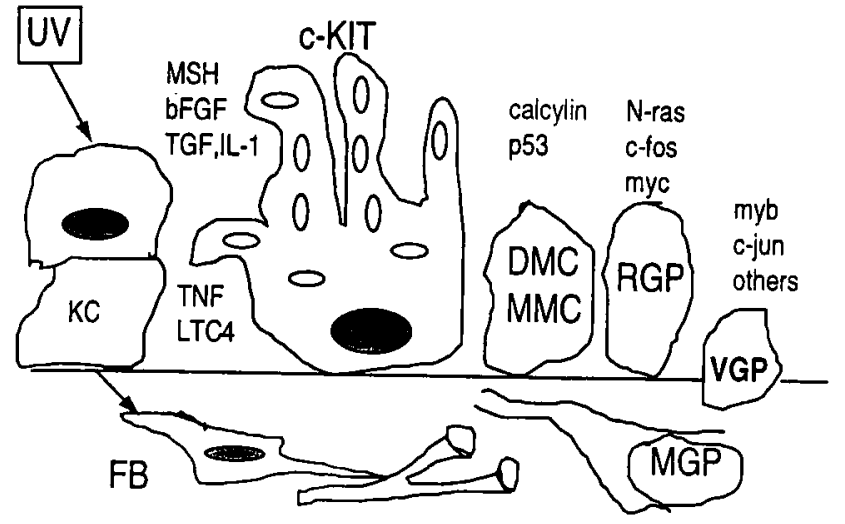

stimulating hormone increases the production of melanosomes as well as eumelanin pigments. EPR (Electron Paramagnetic Resonance) studies have provided evidence for the presence of semiquinone radicals $\left(\mathrm{QH}^{-\bullet} / \mathrm{Q}^{-\bullet}\right)$ in eumelanin ${ }^{11}$ and our own studies have shown that the

Fig 1 Growth factors and regulation in cascade of melanocyte differentiation and transformation UV can be the most potent stimulator for melanogenesis. It can stimulate the production of $\mathrm{MSH}$ (mclanocyte stimulating hormone) in the keratinocyte and upregulate the $\mathrm{MSH}$ receptor on the melanocyte, which would then be stimulated to produce new melanin pigment through cyclic AMP cascade. It can also affect the transformation of melanocytes and dysplastic melanocyte (DMC) which would then follow the step-wise growth phases from radical growth phase (RGP) to vertical growth phase (VGP) and finally metastatic growth phase (MGP). Fibroblasts (FB) in the dermis can also produce the melanocyte differentiation and growth factor (e.g. basic fibroblast growth factor: bFGF).<smiles>CCCCC(N)Cc1ccc(O)cc1</smiles>

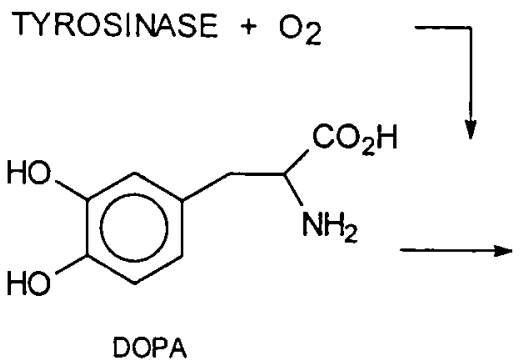<smiles>NC(CC1=CC(=O)C(=O)C=C1)C(=O)O</smiles>

DOPAQUINONE<smiles>O=C1C=C2CC(C(=O)O)NC2=CC1=O</smiles>

DOPACHROME<smiles>O=C(O)C1Cc2cc(O)c(O)cc2N1</smiles>

LEUCODOPACHROME

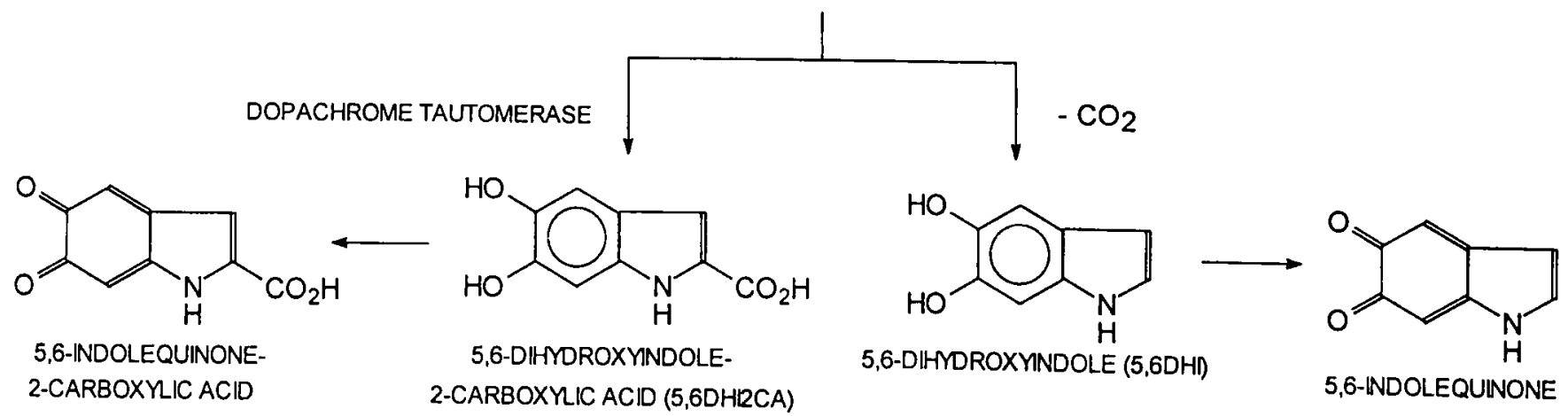
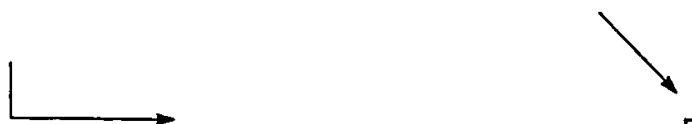

\section{EUMELANIN}

Fig 2 Metabolic and chemical reactions of cumelanin synthesis. 


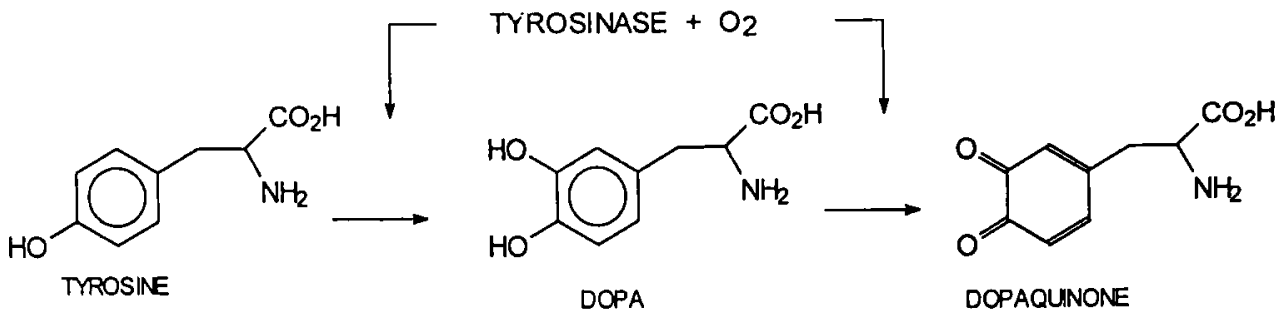

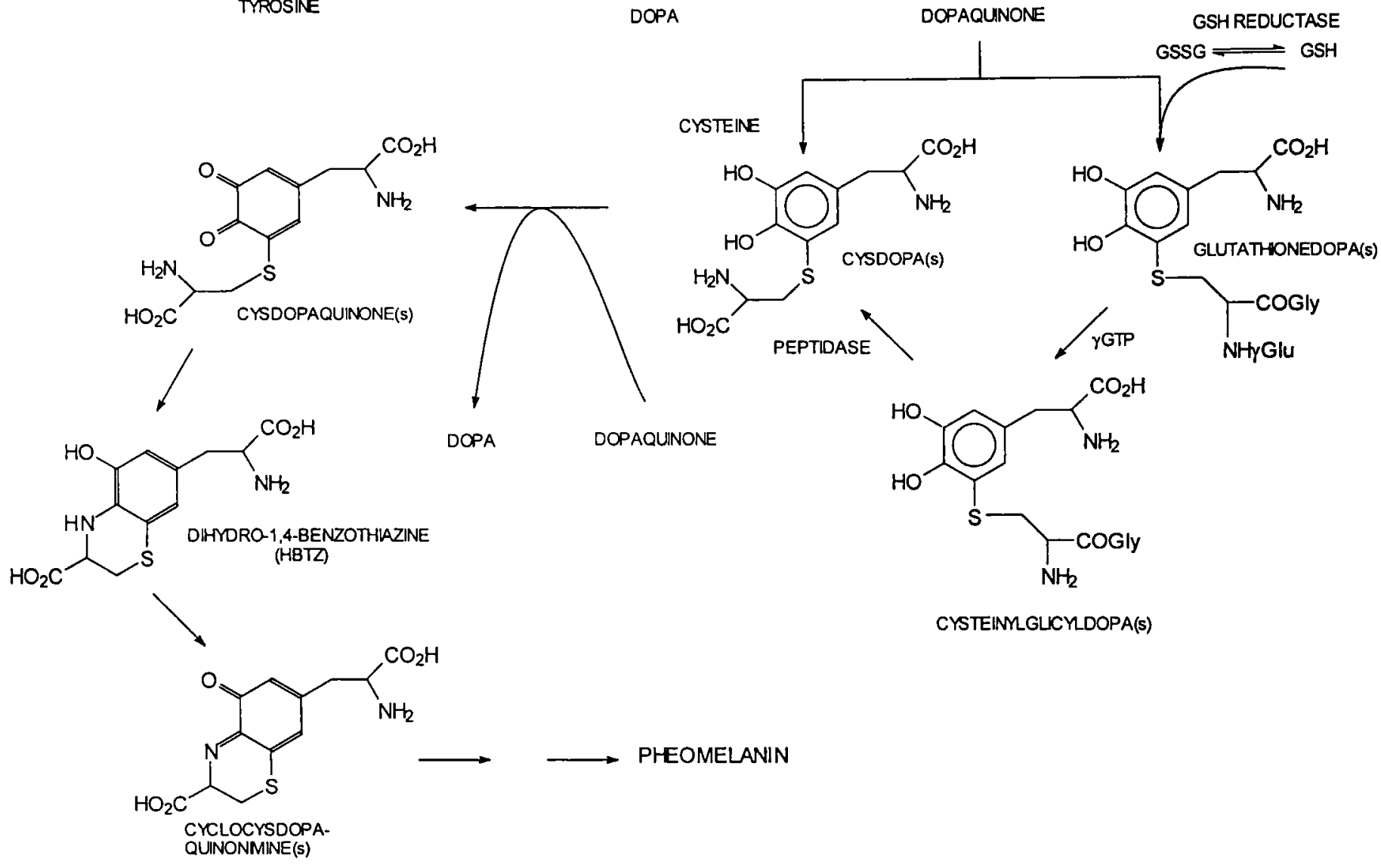

Fig 3 Metabolic and chemical reactions of pheomelanin synthesis.

concentration of the semiquinone radicals increases on irradiation $(>300 \mathrm{~nm}) .^{2}$ While it is not clear whether these melanin radicals are cytotoxic or not, they could participate in known physiologically harmful free radical reactions (Fig 4).

Melanins have been shown to produce $\mathrm{O}_{2}{ }^{-\bullet}$ on irradiation. ${ }^{12}$ Excessive production of $\mathrm{O}_{2}^{\bullet}, \mathrm{H}_{2} \mathrm{O}_{2}, \mathrm{OH}^{-\bullet}$ and $\mathrm{Fe}^{2+}$ could lead to cell damage. However, the contribution of these reactions to cytotoxicity is likely to be minimal because these reactions are very slow at acidic $\mathrm{pH}$ and melanosomal $\mathrm{pH}$ is in the range of $3.0-4.5 .^{13}$ Despite this, there are reports showing cytotoxic effects for eumelanin and pheomelanin. ${ }^{14}$ In these experiments, melanin added to the cell culture medium and taken up by the cell might end up in the cytosol and other subcellular compartments where conditions $(\mathrm{pH})$ are likely to be more favourable for the above damaging free radical reactions.

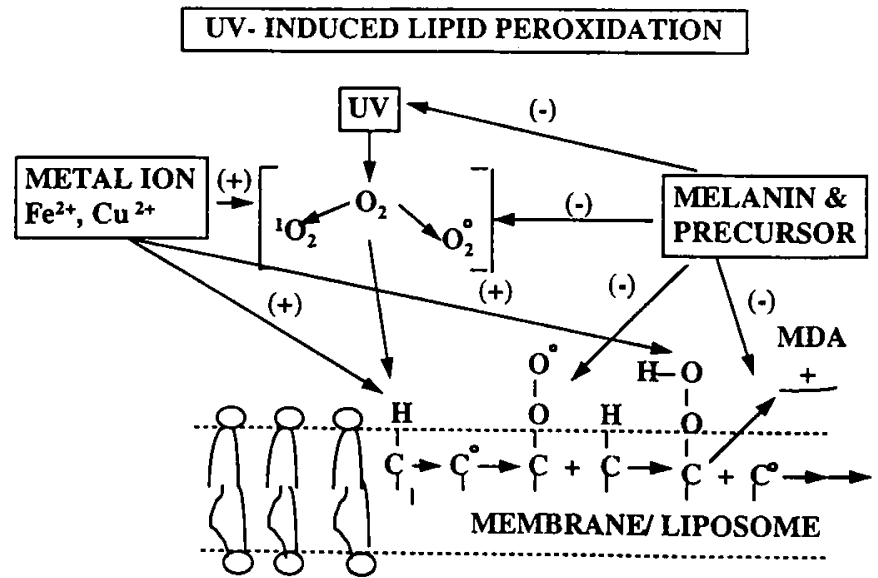

Fig 4 UV-induced cell damage by lipid peroxidation of the cell membrane. Melanin pigments and their precursors, in particular, pheomclanin and its precursor intermediate, 5-S-cysteinyldopa can become pro-oxidant causing lipid peroxidation in the presence of metal ions, e.g. $\mathrm{FE}^{2+}, \mathrm{Fe}^{3+}$ and $\mathrm{Cu}^{2+}$ after exposure to UVR. 
Photoprotective vs phototoxic actions of melanin pigments

In order to study the photoprotective and phototoxic actions of melanin pigments, the experimental conditions mimicking melanosomes were prepared artificially by using soy bean phosphatidylcholine liposomes. The lipid peroxidation in UV-irradiated liposomes were measured as a means to examine the primary membrane events after exposure of melanins and their precursors to UV light. ${ }^{15,16}$ Liposomes were made from phospholipids containing unsaturated fatty acyl chains which are vulnerable to oxidative damage. Two stages of lipid peroxidation were measured in this study. The early lipid peroxidation stage, conjugated dienes by UV absorption and the production of hydroperoxides by iodometry and tetramethylbenzidine (TMB), and the late lipid peroxidation stage, formation of malonedialdehyde (MDA) by HPLC and thiobarbituric acid reactive substances (TBARS) by spectrophotometry were examined in the presence of various sources of melanins, including eumelanin and $\mathrm{DHI}$ as well as pheomelanin and SCD.

Both eumelanin and pheomelanin were effective in decreasing UVR-induced lipid peroxidation product, with pheomelanin being more effective than eumelanin for all three methods of detection, i.e. conjugated dienes, iodometry and TMB. Our preliminary experiment measuring lipid peroxidation has also shown that natural squid eumelanins have anti-lipid peroxidation properties similar to those of three different synthetic eumelanins made from dopa oxidation. The greater anti-oxidant activity of pheomelanin over eumelanin may partly be a reflection of the fact that the former is completely dissolved while the latter remains as a suspension during irradiation. The melanin precursors, DHI and SCD, were even more effective than the mature pigments, DHI having more significant antioxidant activity than SCD. ${ }^{17}$

The decrease in lipid peroxidation occurred, not only in the early stage of lipid peroxidation product such as conjugated dienes and lipid hydroperoxide, the formation of which might be rapidly reversible in vivo and in vitro, but also in the potentially more carcinogenic late-stage product such as TBARS. Pheomelanin was an effective antioxidant at concentrations as low as $5 \mu \mathrm{g} / \mathrm{ml}$. In contrast, eumelanin showed no antioxidant activity when late-stage products were measured. Eumelanin precursor, DHI, showed the highest antioxidant property in latestage lipid peroxidation. The ability of pheomelanin, its precursor, SCD, and eumelanin precursor, DHI, to decrease the formation of late-stage lipid peroxidation product is an indication of their participation in preventing the initiating species or the breaking of chain reaction products involved in lipid peroxidation. The TBARS method is applicable in the presence of iron and this has allowed us to characterize some potentially harmful properties of melanin and their precursors. The addition of a comparatively high concentration of ferric iron during UVR exposure, we changed the oxidative environment of the pigment and lipids. Ferric iron is known to be present within the melanosomal compartment. Metal iron and its ionic forms are also known to increase in keratinocytes after UVR exposure. ${ }^{18,19}$ The presence of ferric iron caused the mature pigment to switch from functioning as an antioxidant membrane protector to membrane damaging pro-oxidant when exposed to UVR. This effect was reversed in the presence of EDTA which chelates the iron. In the presence of ferric iron at low concentration, SCD also showed the highest pro-oxidant effect. Importantly, however, DHI did not show any pro-oxidant activity at both high and low concentrations of ferric iron and remained as an effective antioxidant. Under pro-oxidant conditions of all tested pigments, pheomelanin was found to be the most potent inducer of late-stage peroxidation product. One can, therefore, speculate that membrane-damaging potential of pheomelanin and SCD under pro-oxidative conditions would make them hazardous if these conditions arise in vivo such as after heavy intermittent exposure to UVR during summer.

\section{Perturbed melanin synthesis and increased UVR sensitivity in possible melanoma precursor lesions: Familial atypical multiple moles and melanoma syndrome}

To further evaluate the possible functional role of melanin pigmentation, the amount of eumelanin and pheomelanin was directly measured in the tissue of dysplastic melanocytic nevi (or atypical moles) because patients suffering from familial atypical multiple moles and melanoma (FAMMM) or previously called familial dysplastic melanocytic nevus syndrome possess a high risk for melanoma development as much as $80 \%$ to $100 \%$ in their lifetime. ${ }^{20.21}$ In these syndrome patients, there is an increased susceptibility to UV-induced somatic mutation such as enhanced cell killing upon UV exposure or altered DNA repair and the abnormal DNA synthesis which is, however, distinct from that of XP variant cell lines. ${ }^{22}$

The pheomelanin content of common melanocytic nevi was compared to atypical moles (previously called dysplastic melanocytic nevi)..$^{23}$ The data clearly showed an increase in the amount of pheomelanin in dysplastic melanocytic nevi as measured by AHP, a pheomelanin indicator, and PTCA, a eumelanin indicator.

The direct measurement of tissue content of eumelanin and pheomelanin in atypical moles indicates that there may be perturbed melanogenesis in patients with this syndrome. Furthermore, it was shown that melanosomes in atypical nevocytes were oval in shape and contained granular material similar to that seen with pheomelano- 
somes. ${ }^{24}$ The recent study from several research groups also indicated that there is an increased level of pheomelanin precursor, $\mathrm{SCD}$ in these atypical moles and there is an increased blood level of SCD as well as other melanin metabolites after UVR in melanoma patients. ${ }^{25-27}$ All these findings indicate that pheomelanin and its precursor, $S C D$, in the presence of UVR may be harmful in certain patients such as FAMMM syndrome patients who have a high risk of melanoma development.

\section{Melanogenesis and Functional Interaction of Tyrosinase and Lamp Gene Families}

\section{Major genes and their products involved in melanogenesis} cascade

Three genetic loci are of the greatest importance in the melanin synthesis pathway, and most extensively studied in laboratory mice. These are the $c$ (color)-locus for tyrosinase, the a (agouti)-locus for amounts and distribution of pheomelanin and eumelanin within the integument, and the $b$ (brown)-locus for the type of eumelanin synthesized by melanocytes. ${ }^{28.29}$ The level of tyrosinase mRNA and tyrosinase is elevated in brown $(b / b)$ melanocytes suggesting an influence of the $b$ locus on the transcription of the tyrosinase gene..$^{30}$ The $b$-locus deletions or null point mutations do not eliminate pigmentation, but result in mice with brown, instead of black eumelanin. ${ }^{31}$ The $b$-locus also codes for a protein forming part of the melanosomal matrix. This b-locus protein possesses $36 \%$ amino acid homology to tyrosinase, and has been termed tyrosinase-related protein-1 (TRP-1). Other mutations at this locus result in an intermediate phenotype between black and brown, due to a reduction in the amount of TRP-1 mRNA, whilst still others have a dominant phenotype resulting in premature melanocyte death. ${ }^{32}$ This gene has been initially isolated as a candidate gene for tyrosinase by immunoscreening of a melanocytes cDNA expression library using anti-tyrosinase antibody (TMH-1). When the same melanocyte cDNA library was immunoscreened by the same anti-tyrosinase antibody, another cDNA was identified. This clone showed some cross hybridization with a TRP-1 cDNA, but encoded a different protein (hence called TRP-2). The protein was found to have a dopachrome tautomerase activity and its human cDNA has been cloned recently ${ }^{33}$ and mapped to mouse $s$ (slaty-slt) locus. ${ }^{38.41}$

Functional interaction of tyrosinase and LAMP gene families after exposure to $U V R$

Human tyrosinase and LAMP-gene families share many common structural and biological properties. In fact, melanosomes and lysosomes are known to possess many similarities and could derive from common precursor vacuoles (Fig 5). Human LAMP-1 and LAMP-2 sequences reveal strong homology between the two molecules, particularly in the proximity to the $\mathrm{COOH}$-cytoplasmic terminal end. It is suggested that this portion is important for targeting the molecules to lysosomes. ${ }^{34,35}$ Similar to the LAMP molecule, tyrosinase and TRPs possess a single transmembrane domain, and the cytoplasmic tails at $\mathrm{COOH}$ terminal show some common features such as motif with basic amino acids and putative tyrosine phosphorylation sites. ${ }^{36,37}$ These structural characteristics may contribute to the activation of melanogenesis-associated proteins by allowing them to target to the melanosomal membrane, and this targeting signal could be regulated by the tyrosine phosphorylation-dephosphorylation of cytoplasmic tails. Importantly, tyrosinase and TRP-1, possesses two putative tyrosine phosphorylation sites which are common, whereas LAMP-1, LAMP-2 and TRP-2 show only one putative tyrosine phosphorylation site, but this site is identical or similar to one of the two putative tyrosine phosphorylation sites seen in tyrosinase and TRP-1. Therefore, there may be some common functional properties between tyrosinase and LAMPgene families or some structural interaction between these two melanogenesis-associated gene families. Human melanoma cells and normal melanocytes increase the expression of tyrosinase and Lamp-gene families and their gene products after exposure to UVR. ${ }^{38}$

The findings of increased melanin synthesis in pigmented melanoma cell lines and the absence of melanin synthesis in amelanotic melanoma cells after exposure to UVR were in parallel to those findings of semi-quantitative RT-PCR analysis of mRNA expression of tyrosinase, TRP-1, LAMP-1 and LAMP-3 in these melanotic and

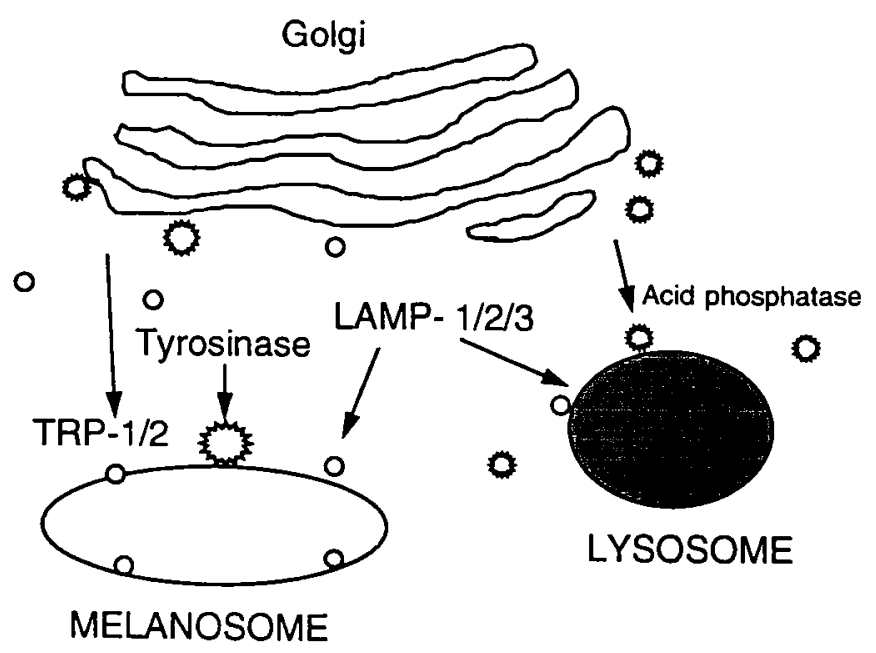

Fig 5 Expression of tyrosinase and LAMP gene family products in melanosomes and lysosomes. LAMP gene family is expressed in both the melanosome and lysosome. 
amelanotic cell lines after exposure to UVB for seven consecutive days. There is an increased expression of tyrosinase in pigmented melanoma cells, e.g. G361 which is followed by an increased expression of TRP-1. There is no tyrosinase gene expression in amelanotic melanoma cells, e.g. SK-MEL-24 before and after UVB exposure, which is again followed by the negative expression of TRP-1 in the same cell line. Interestingly, certain amelanotic melanoma cell lines, e.g. C32 showed the presence of increased tyrosinase gene expression which was, however, not associated with TRP-1 expression. It appears, therefore, that the proper melanin biosynthesis within the melanocyte may require the coordinated mRNA expression of both tyrosinase and TRP-1. In contrast, there was no alteration in the expression of LAMP-3 which appears to be more related to the structural component of the lysosomal or melanosomal membrane.

Functional interaction of tyrosinase and LAMP gene families after tyrosinase gene family transfection

In order to further study the functional interaction of LAMP and tyrosinase-gene families in melanogenesis, three major experiments have been carried out recently by our research group. These include; (a) co-transfection of tyrosinase and TRP-1 into amelanotic melanoma cells, (b) treatment of melanotic melanoma cells with antisense oligodeoxynucleotides against LAMP-1, and (c) subcellular distribution of tyrosinase/TRP-1 and LAMP-1/ LAMP-3.

For the co-transfection of human tyrosinase and TRP1 genes, human amelanotic melanoma cells of SK-MEL24 and C32 as well as COS7 monkey kidney cells were used. C32 has tyrosinase gene expression but there is no actual melanin synthesis and no TRP-1 gene expression. In contrast, SK-MEL-24 lacks both tyrosinase and TRP-1 gene expression. After co-transfection of human tyrosinase cDNA, there was an increased tyrosinase activity as well as melanin synthesis. In contrast, TRP-1 transfection did not show any such increase of tyrosinase activity and melanin synthesis, indicating that TRP-1 itself is not responsible for direct activation or synthesis of tyrosinase as well as melanin pigments. However, it may help tyrosinase-transfected cells to protect themselves from programmed cell death due to tyrosinase-mediated toxicity. This hypothesis was further substantiated by our finding that melanoma cells and COS-7 cells transfected with human tyrosinase gene alone revealed many vacuolations, and even premature death of cells, indicating that human tyrosinase gene product itself is toxic to the cells. In contrast, co-transfection with human tyrosinase and TRP-1 genes showed the production of many oval granules which were positive with anti-TRP-1 antibody immunostaining and contained melanized melanosomal matrix under electron microscopic observation. There was no vacuolation as was seen in cells with single transfection of human tyrosinase gene. TRP-1 transfection alone did not influence directly any melanin synthesis. There was no increase of tyrosinase nor was there any increase of melanin synthesis. ${ }^{39,41}$

To further investigate the functional interaction between tyrosinase and LAMP-family genes, comparative PCR analyses were carried out using specific primers targeting Lamp-family genes, including LAMP-1, LAMP-2 and LAMP-3 as well as tyrosinase and TRP-1 cDNAs. Expression of LAMP-3 was detected in all cells treated with either individual transfection of human tyrosinase or TRP-1 gene or co-transfection of both. No significant variations were observed among the individual experimental groups. In contrast, enhanced mRNA expression of LAMP-1 gene was observed in human tyrosinase and TRP-1 co-transfected cells, but not in individual transfectants. These observations were shown by the protein expression study using Western blotting. The results may indicate that there is a coordinated upregulation of LAMP-1 gene and protein synthesis by cotransfection of tyrosinase and TRP-1 cDNAs, but not by a single transfection of either one of them. This hypothesis was further tested by the antisense LAMP-1 gene hybridization experiment. In this experiment, human melanoma cells of G361 were incubated with antisense oligodeoxynucleotide targeted on LAMP-1 gene in culture. They were then processed for immunoprecipitation using 3 different monoclonal antibodies against human TRP-1 such as HMSA-5, 3B5 and 2G10. This antisense experiment has clearly shown significant and coordinated decrease in the expression of TRP-1 in G361 melanoma cells which were hybridized with anti-LAMP-1 oligodeoxynucleotide. TRP-1 is an N-linked glycoprotein, having approximately 6 to 8 oligosacharides after $\mathrm{N}$-glycanase digestion. Therefore, human TRP-1 which was originally $69 \mathrm{kDa}$ in size moved approximately to $50 \mathrm{kDa}$ after $\mathrm{N}$-glycanase digestion. TRP-1 does not, however, have any O-linked oligosaccharides.

In order to characterize the glycosylation process of human TRP-1, cells were treated with tunicamycine which inhibits all the processes of glycosylation and monensine which inhibits the glycosylation process at the site of medial portion of the Golgi complex. In cultured human cells such as normal human melanocytes and melanoma cells treated with monensine, there was a remarkable increase in the expression of TRP-1 as compared to control. In contrast, almost complete loss of TRP-1 expression was seen after treatment of tunicamycin (Fig 6). ${ }^{+1}$ The findings clearly indicated that TRP. 1 is transferred from ER to the medial portion of the Golgi complex where full glycosylation may be completed. On the other hand, tyrosinase will be processed during glycosylation to the periphery of the Golgi network, trans-Golgi network, and then subsequently transferred 


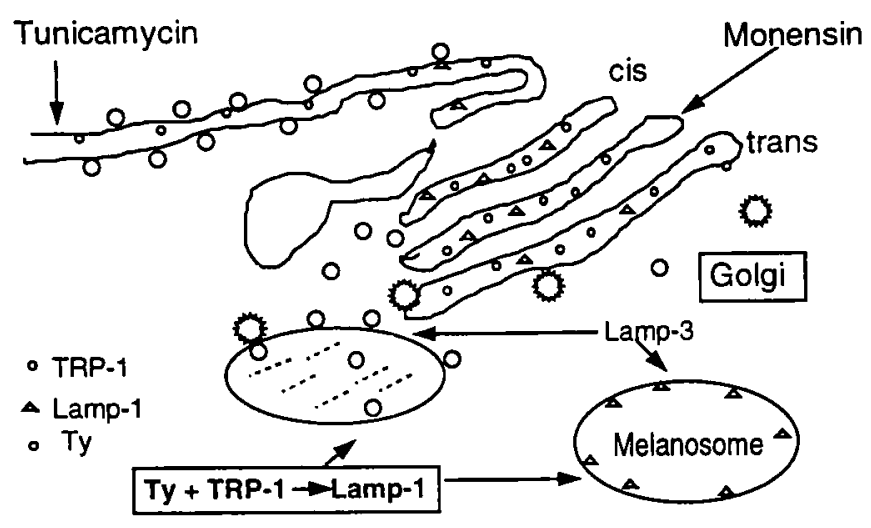

Fig 6 Coordinated interaction of tyrosinase (Ty) TRP-1 and LAMP-1. Inhibition of glycosylation of tyrosinase and TRP-1 by tunicamycin and monensin. When melanoma cells are cxposed to monensin. which perturbs the transport of glycoproteins at the site of medial Golgi, there was an increased expression of TRP-1. Tunicamycin, which inhibits the glycosylation of glycoproteins at the site of ER (cndoplasmic reticulum), inhibited completely the expression of TRP-1 and tyrosinase.

to the melanosomes.

Under electron microscopic immunogold study of HMSA-5, which is identical to TRP-1, immunogold particles were labelled on the medial portion of the Golgi complex as well as small vesicles but not coated vesicles in the vicinity of Golgi complexes. ${ }^{42}$ Importantly, the same immunogold positive vesicles were seen on the surface of secondary lysosomes. Specifically, HMSA-5 positive immunogold particles were seen along the outer membrane of the melanosomes. They were accumulated on the surface of melanosomal membrane in the early melanosomal maturation, such as in stages 1 and 2 . The size of individual microvesicles or immunogold positive vesicles ranged approximately from $40 \mathrm{~nm}$ in diameter. These small vesicles were present in various stages of melanosomal maturation, and located along the surface of the melanosomal membrane. When they were present in mature stage 4 melanosomes, they often revealed the electronlucency. At this stage, HMSA-5 expression was negative because the antigenic epitope of HMSA-5 appeared to be embedded in the highly melanized melanosomal matrix. ${ }^{42}$

\section{Importance of glycosylation for proper melanization within melanosomal compartment}

When immunoelectron microscopic study was done on immunogold-particle distribution reacted with antiHMSA-7, which is relevant to LAMP-3 or CD63, and the immunogold particles were located, similar to HMSA-5, within the small vesicles, associated with melanosomal membrane. This was further confirmed by anti-LAMP-3 immunoelectron micrograph using LS62, indicating that LAMP-3 is present in both melanosomes and lysosomes.

In contrast, tyrosinase is present in the trans-Golgi network and the coated vesicles and melanosomes in late stages of 2, 3, and 4. Importantly, there are a fairly large number of small vesicles present in the vicinity of Golgi complexes, but none of these small vesicles are positive with tyrosinase activity. The structure and distribution pattern of these small vesicles were identical to those of HMSA-5 (TRP-1) or HMSA-7 (LAMP-3) molecules. Immunoelectron microscopic and cytochemical studies indicate that TRP-1 and probably also LAMP-gene family proteins, take the subcellular localization different from that of tyrosinase in the Golgi complex. One may then wonder why TRP-1 and LAMP gene families need to have the subcellular distribution different from that of tyrosinase before they are incorporated into melanosomes and what the functional interaction is present between TRP-1 and LAMP gene family during melanization.

This question may be better answered when one compares the structural properties of tyrosinase and Lampgene family products. TRP-1 is an N-linked glycoprotein which has only 6 to $8 \mathrm{~N}$-linked oligosaccharides but no O-lined oligosaccharides. In contrast, LAMP-1 and LAMP-2 are of similar molecular size and have both $\mathrm{N}$-linked and $\mathrm{O}$-linked oligosaccharides, indicating that LAMP-1 and LAMP-2, but not LAMP-3 are highly glycosylated as compared to TRP-1. It is known that in lysosomes, the LAMP-1 and LAMP-2 coat the inner surface of the lysosomes, serving as a barrier to prevent the action of lysosomal hydrolases on the membrane.

Similar to lysosomes, LAMP-1 and probably LAMP-2 molecules which are up-regulated after co-transfection of tyrosinase and TRP-1, are present on the inner surface of melanosomal membrane, functioning as a scavenger of free radicals and other free radical products which are produced during new melanogenesis through tyrosinase activation. This process, however, does not appear to be activated by either tyrosinase or TRP-1 alone. In other words, tyrosinase and TRP-1 coordinate together to upregulate LAMP-1 which will then be transferred to the melanosomes, coating the inner surface of the melanosomal membrane. If this speculation were true, then LAMP-1, not TRP-1, is a key molecule which directly protects the melanosomal membrane against tyrosinasemediated membrane damage, by having a high content of $\mathrm{N}$ - and $\mathrm{O}$-linked oligosaccharides. In contrast, TRP-1 has only a small number of $\mathrm{N}$-linked oligosaccharides and no O-linked oligosaccharides, so that it can hardly function as a barrier for tyrosinase-mediated oxidative stress within the melanosomal compartment (Fig 7). ${ }^{37}$

Acknowledgements: The work presented in this review article is the product of group efforts. The individual contributants include Stefan Schmitz, Panakkezhum Thomas, Thomas Salopek, Koji Yamada, 


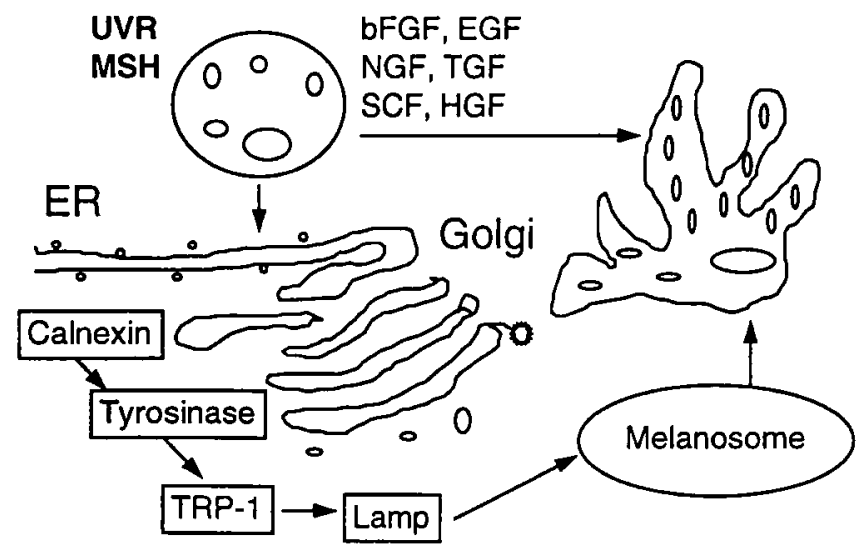

Fig 7 Possible functional interaction of tyrosinase and LAMP genc family products. Calnexin would be a commander of glycosylation of the intermediate molecules of these gene products. Upon the coordination of tyrosinase and TRP-1, LAMP-1 will be upregulated, coating the inner surface of melanosomal membrane which would protect the membrane damage of melanosomal compartment resulting from tyrosinase-mediated oxidation.

Hiroyuki Hara, Dong Luo, Hua Chen, Theresa Allen, Frank Alena, Arihito Ota, Essam Shetta, Krzysztof Reszka and Mu Yong Lee. The author wishes to thank all of their contributions. The work was supported by the Medical Research Council of Canada (PA-1193), the National Cancer Institute of Canada (\#4145), the Alberta Cancer Board (\#R1-91), and the Alberta Heritage Foundation Medical Scientist Award.

\section{References}

1. Jimbow K, Quevedo WC Jr, Fitzpatrick TB, Szabo G: Biology of melanocytes. In: Fitzpatrick TB, Eisen AZ, Wolff K, Freedberg IM, Austen KF, eds, Melanocytes in Dermatology in General Medicine, Vol 1, New York, McGraw Hill, 1993, 261-289

2. Reszka K, Jimbow K: Electron-donor and acceptor propertics of melanin pigments in the skin. In: Fuchs $J$, Packer L, eds, Oxidative Stress in Dermatology, New York, Marcel Dekkcr Inc, 1993. $287-320$

3. Jimbow K, Salopek TG, Dixon WT, Searles GE, Yamada K: The epidermal melanin unit in the pathophysiology of malignant melanoma. Am J Dermatophatol 1991, 13: 179-188

4. Jimbow K, Fitzpatrick TB, Wick M: Biochemistry and physiology of melanin pigmentation. In: Goldsmith LA, cd, Physiology and Molecular Biology of the Skin, Vol 2, New York, Oxford University Press, 1991, 873-909

5. Norris DA, Horikawa T, Morelli JG: Melanocyte destruction and repopulation in vitiligo. Pigment Cell Res 1994, 7: 193-203

6. Jimbow K, Szabo G, Fitzpatrick TB: Ultrastructure of giant granules (macromelanosomes) in the cutaneous pigmented macules of neuro-fibromatosis. J Invest Dermatol 1973, 61: 300-309

7. Halaban R, Kwon BS, Ghosh S, Delli Bori P, Baird A: bFGF as an autocrine growth factor for human inclanosomas. Oncogenes Res 1988, 3: 177-186

8. Bolognia J, Murray M, Pawlek J: UVB-induced melanogencsis may be mediated through the MSH-receptor sy'stem. J Invest Dermatol 1989, 92: 651-656

9. Jimbow K, Andreassi L, Halaban R. Houghton A, Parsons P, Lipkin G: Growth factors and regulators of malignant mclanoma.
In: Cascinelli $O$, Santinami M, Veronesi U, eds, Malignant Melanoma, 1990, 25-38

10. Fusakaw Y, Boulton T, Cobb M, Yarden Y, Fian B, Lyman SD, William SDE, Anderson DM, Zakut R. Mishima Y, Halaban R: c-Kit-kinase induces a cascade of motion tyrosine phosphorylation in normal human melanocytes in response to mask cell growth factor and stimules mitogen-activated protein kinase but is downregulated in melanomas. Mol Biol Cell 1992, 3: 197-209

11. Hill HZ, Hill GL: Eumclanin causes DNA strand breaks and kills cells. Pigment Cell Res 1987, 1: 163-170

12. Korytowski W, Pilas B, Sarna T, Kalyanaraman B: Photoinduced generation of hydrogen peroxide and hydroxyl radicals in melanins. Photochem Photobiol 1987, 45: 185-190

13. Oikawa A, Nakayasu M: Quantitative measurement of melanin. as tyrosinase equivalents and as weight of purified melanin. Yale J Biol Med 1973, 46: 500-507

14. Urabe K. Aroca P. Tsukamoto K, Mascagna D, Palumbo A, Prota G, Hearing VJ: The inherent cytotoxicity of melanin precursors: a revision. Biochim Biophys Acta 1994. 1221: 272-278

15. Schmitz S, Mirzayans R, Jimbow K. Iwashina T, Allen TM: Aborted apoptosis - a possible link between melanoma, melanin pigment phenotype and UV cxposure. J Invest Dermatol 1993. 100: 494

16. Schmitz S, Allen TM, Jimbow K: Polyethylene glycol-mediated delivery of liposome-entrapped pigments into fibroblasts: cxperimental pigment cells as models for mutator phenotypes. Cancer Res 1992, 52: 6638-66+5

17. Schmitz S, Thomas PD. Allen TM, Poznansky MJ, Jimbow K: Dual role of melanin precursors as photoprotective and phototoxic agents: inhibition of ultraviolct radiation-induced lipid peroxidation. Photochem Photobiol 1994 (submitted)

18. Bisset DL, Chatterjcc R, Hannon DP: Chronic ultraviolet radiation-induced increase in skin iron and the photoprotective effect of topically applied iron chelators. Photochem Photobiol 1991, 54: 215-223

19. Aubailly M, Santus R, Salmon S: Ferrous iron release from ferritin by ultraviolet-A radiations. Photochem Photobiol 1991 , 54: $769-773$

20. Perera MI, Um KH, Greene MH, Waters HL, Bredberg A, Kramer KH: Hereditary dysplastic nevus syndrome: lymphoid cell ultraviolet hypermutability in association with increased melanoma susceptibility. Cancer Res 1986, 46: 1005-1009

21. Jimbow K, Lauzon GJ; Malignant mclanoma: a life threatening skin cancer affecting young pcoplc - its diagnosis and management. Ann Royal Col Phys Surg 1990, 23: 105-110

22. Smith PJ, Green MH, Adams D, Paterson MC: Abnormal responses to the carcinogen +-nitroquinoline 1-oxide of cultured fibroblasts from patients with dysplastic nevus syndrome and hereditary cutancous malignant melanoma. Carcinogenesis 1983. 4: $911-916$

23. Salopek TG, Yamada K. Ito S, Jimbow K: Dysplastic melanocrtic nevi contain high levels of pheomelanin: quantitative comparison of phcomelanin/eumelanin levels between normal skin, common nevi and dysplastic nevi. Pigment Cell Res 1991, 4: 172-179

24. Takahashi H, Horikoshi T, Jimbow K: Finc structural characterization of melanosomes in dysplastic nevi. Cancer 1985. 56: 111123

25. Hansson C, Edholm LE, Agup G, Rosman H, Rosengen AM, Rosengren E: The quantitative determination of 5-S-cjsteinyldopa and dopa in normal scrum and in scrum from paticnts with malignant melanoma by mcans of high-pressure liquid chcmotherapy. Clin Chem Acta 1978. 88: 419- +27

26. Peterson LL, Woodward WR, Fletcher WS, Palmquist M. Tucker MA, Ilias A: Plasma 5-S-cysteinyldopa differentiatcs patients with primary and metastatic melanoma from paticnts with dys- 
plastic nevus syndrome and normal subjects. J Am Acad Dermatol 1988, 19: 509-511

27. Hara H, Yamada K, Jimbow K, Walsh N: High plasma level of a eumclanin precursor, 6-hydroxy-5-mcthoxyindole-2-carboxylic acid as a new prognostic marker for malignant melanoma. J Invest Dermatol 1994, 102: 501-505

28. Kwon BS, Haq AK, Pomerantz SH, Halaban R: Isolation and sequence of a cDNA clone for human tyrosinase that maps at the mouse c-albino locus. Proc Natl Acad Sci USA 1987, 84: 74737477

29. Shibahara S: Functional analysis of the tyrosinase gene and brown-locus protein gene promoters. J Invest Dermatol 1993 , 100: $146 \mathrm{~S}-149 \mathrm{~S}$

30. Jackson IJ, Chambers DM, Budd PS, Johnson R: The tyrosinaserelated protein-1 gene has a structure and promoter sequence very different from tyrosinase. Nucleic Acids 1991, 19: 37993804

31. Takeuchi T, Tamaka S, Tamaka M: Expression of tyrosinase gene in transgenic mice: programmed versus non-programmed expression. J Invest Dermatol 1993, 100: 1+1S-145S

32. Bennett DC: Genetics, development and malignancy of melanocytes. Int Rev Cytol 1993, 146: 191-260

33. Yokoyama K, Yasumoto K, Suzaki H, Shibahara S: Cloning of the human DOPA chrome tautomerase/tyrosinase-related protein-2 gene and identification of two regulating regions required for its pigment cell-specific expression. J Biol Chem 1994, 269: 27080-27087

34. Carlsson SR, Roth J, Piller F, Fukuda M: Isolation and characterization of human lysosomal membrane glycoprotein, h-lamp-1 and h-lamp-2, major sialoglycoproteins carrying polylactosaminoglycan. J Biol Chem 1988, 263: 18911-18919

35. Barrio-Canal JG, Bonifacino JS, Yuan L, Sandoval IV: Biosyn- thesis, glycosylation, movement through the Golgi system, and transport to lysosomes by $\mathrm{N}$-linked carbohydrate-independent mechanism of three lysosomal integral membrane proteins. J Biol Chem 1986, 261: 16755-16763

36. Williams MA, Fukuda M: Accumulation of membrane glycoproteins in lysosomes requires a tyrosine residue at a particular position in the cytoplasmic tail. J Cell Biol 1990, 111: 955-966

37. Jimbow K, Hara H, Vinayagamoorthy T, Luo D, Dakour J, Yamada K, Dixon W, Chen H: Molecular control of melanogenesis: functional assessment of tyrosinase and Lamp gene families by UV exposure and gene co-transfection, and cloning of a cDNA encoding calnexin, a possible melanogeneis "chaperon". J Dermatol 1994, 21: 9-21

38. Hara $H$, Lee $M H$, Chen $H$, Luo $D$, Jimbow $K$ : Role of gene expression and protein synthesis of tyrosinase, TRP-1, LAMP-1 and CD63 in UVB-induced melanogenesis in human melanomas. J Invest Dermatol 1994, 102: 495-500

39. Luo D, Chen H, Jimbow K: Co-transfection of genes encoding human tyrosinase and tyrosinase-related protein-1 prevents melanocyte death and enhances melanin pigmentation and gene expression of Lamp-1. Exp Cell Res 1994, 213: 231-241

40. Jimbow K, Luo D, Chen H, Lee MH: Coordinated mRNA and protein expression of human Lamp-1 in new melanogenesis after UV-B exposure and co-transfection of human tyrosinase and TRP-1 cDNAs. Pigment Cell Res 1994, 7: 73-80

41. Der JE, Dixon WT, Jimbow K, Horikoshi T: A murine monoclonal antibody, MoAb HMSA-5, against a melanosomal component highly cxpressed in early stages, and common to normal and ncoplastic melanocytes. Br J Cancer 1993, 67: 47-57

42. Jimbow K, Alena F, Dixon W, Hara H: Regulatory factors of pheo- and eumelanogenesis in melanogenic compartments. Pigment cell Res 1992, 2(Suppl): 36-42 